\title{
Use of the L2C signal for inversions of GPS radio occultation data in the neutral atmosphere
}

\author{
S. V. Sokolovskiy $\cdot$ W. S. Schreiner • \\ Z. Zeng $\cdot$ D. C. Hunt $\cdot$ Y.-H. Kuo $\cdot$ T. K. Meehan $\cdot$ \\ T. W. Stecheson - A. J. Mannucci - C. O. Ao
}

Received: 24 May 2013/ Accepted: 12 September 2013/Published online: 9 October 2013

(C) The Author(s) 2013. This article is published with open access at Springerlink.com

\begin{abstract}
Results from processing FORMOSAT-3/COSMIC radio occultations (RO) with the new GPS L2C signal acquired both in phase locked loop (PLL) and open loop (OL) modes are presented. Analysis of L2P, L2C, and L1CA signals acquired in PLL mode shows that in the presence of strong ionospheric scintillation not only L2P tracking, but also L1CA tracking often fails, while L2C tracking is most stable. The use of $\mathrm{L} 2 \mathrm{C}$ improves current RO processing in the neutral atmosphere mainly by increasing the number of processed occultations (due to significant reduction in the number of L2 tracking failures) and marginally by a reduction in noise in statistics. The latter is due to the combination of reduced L2C noise (compared to L2P) and increased L1CA noise in those occultations where L2P would have failed. This result suggests application of OL tracking for L1CA and L2C signals throughout an entire occultation to optimally acquire RO data. Two methods of concurrent processing of L1CA and L2C RO signals are considered. Based on testing of individual occultations, these methods allow: (1) reduction in uncertainty of bending angles retrieved by wave optics in the lower troposphere and (2) reduction in small-scale residual errors of the ionospheric correction in the stratosphere.
\end{abstract}

Keywords GPS radio occultation - Signal tracking · Inversion methods

S. V. Sokolovskiy $(\bowtie) \cdot$ W. S. Schreiner · Z. Zeng .

D. C. Hunt · Y.-H. Kuo

University Corporation for Atmospheric Research,

Boulder, CO, USA

e-mail: sergey@ucar.edu

T. K. Meehan - T. W. Stecheson - A. J. Mannucci - C. O. Ao Jet Propulsion Laboratory of the California Institute of Technology, Pasadena, CA, USA

\section{Introduction}

To date, GPS radio occultation remote sensing of the neutral atmosphere (Kursinski et al. 1997) has been using two GPS signals: L1CA at $1.57542 \mathrm{GHz}$ and L2P at $1.2276 \mathrm{GHz}$, where the second signal is used for ionospheric correction. While the L1CA signal is open, the L2P signal is encrypted. In FORMOSAT-3/COSMIC (hereafter referred to as F3/C for brevity), L1CA is tracked in a phase locked loop (PLL) mode between the top of an occultation and a transition point corresponding to about $-10 \mathrm{~km}$ height of straight line (HSL) between the GPS and low earth orbiting (LEO) satellite (approximately equal to $10 \mathrm{~km}$ height of ray tangent point (HTP)). L2P is tracked in a semi-codeless PLL mode (aided by L1CA) (Woo 2000; Meehan et al. 2000) between the top of an occultation and the transition point. Below the transition point, L1CA is tracked in the model-aided open loop (OL) mode (Sokolovskiy 2001; Ao et al. 2009; Sokolovskiy and Rocken 2004), while L2P is not tracked. Semi-codeless PLL tracking of L2P results in low signal-to-noise ratio (SNR) and tracking instability in the presence of the ionospheric scintillation, thus making L2P noisy and unusable in some occultations well above the transition point.

Figure 1 shows an example of significant noise (tracking errors) on L2P Doppler at HSL about 30, 80-90, and $100 \mathrm{~km}$ due to localized ionospheric scintillation. Even though in this case the lock on both L1CA and L2P is maintained, the L2P noise results in significant errors of the ionospheric correction. Figure 2 shows an example of an occultation where the effect of a sporadic $\mathrm{E}$ layer at HSL about $90-100 \mathrm{~km}$ results in loss of lock on L2P. Figure 3 shows an example of an occultation where the effect of a sporadic E layer at HSL about $100 \mathrm{~km}$ results in loss of lock on both L2P and L1CA. 


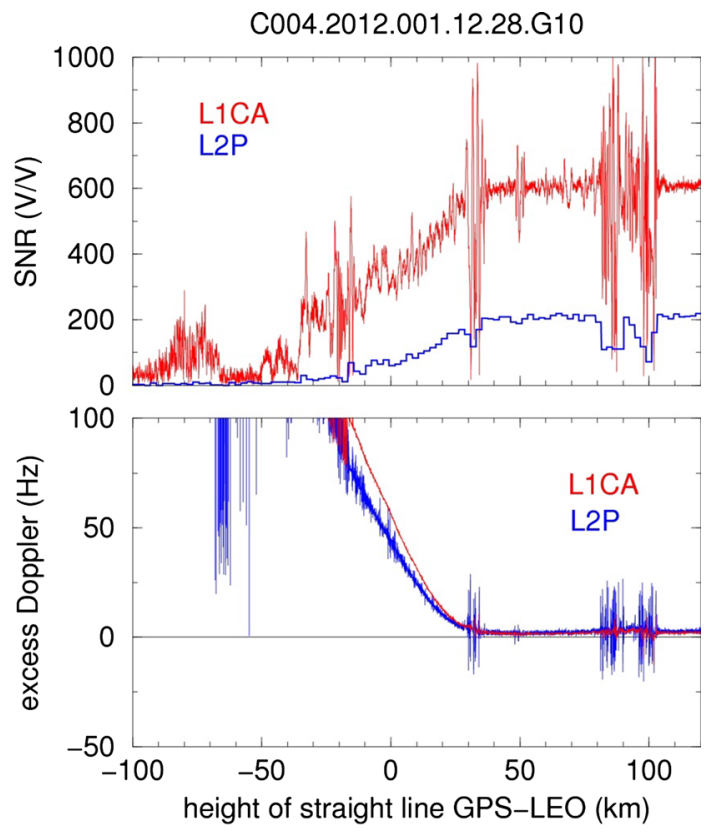

Fig. 1 Example of F3C setting occultation with significant L2P noise induced by ionospheric scintillation. No loss of lock. Upper panel L1CA and L2P SNRs; lower panel excess Doppler values

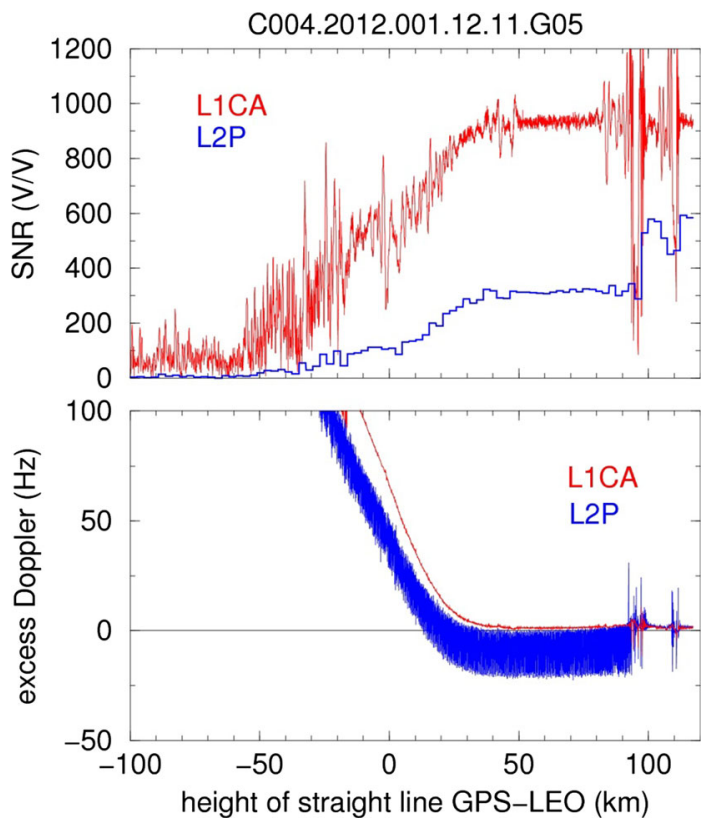

Fig. 2 Example of F3C setting occultation where lock on L2P is lost due to strong ionospheric scintillation. Lock on L1CA is maintained. Upper panel L1CA and L2P SNRs; lower panel excess Doppler values

In the $\mathrm{RO}$ inversion processing at the $\mathrm{F} 3 \mathrm{C}$ Data Analysis and Archive Center (CDAAC), the L1CA-connected phase provided by the RO receiver is not used. The L1CA signal is used as a sequence of complex samples I and Q, which is down-converted with a Doppler model based on positions,

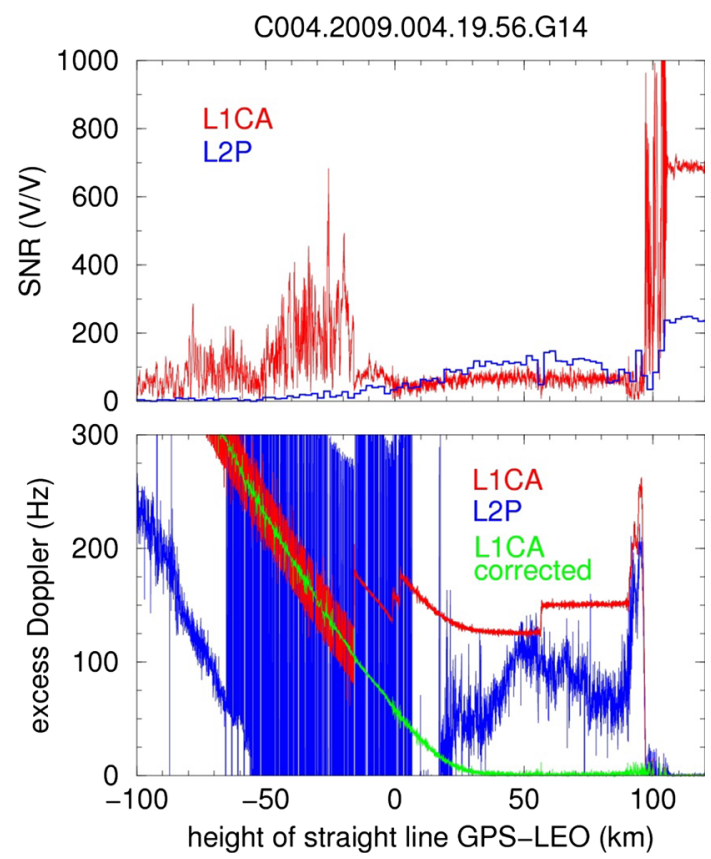

Fig. 3 Example of F3C setting occultation where lock on both L2P and L1CA is lost due to strong ionospheric scintillation. Upper panel L1CA and L2P SNRs; lower panel excess Doppler values; green line shows corrected L1CA Doppler

velocities, and bending angle climatology. Then, the phase is connected by resolving full cycle ambiguities. Additionally, in PLL mode (and in OL mode when data modulation replica is not available), the connection of the phase includes resolving of half-cycle ambiguities. This preprocessing is discussed in details in Sokolovskiy et al. (2009). Since, in most cases, the tracking errors on L1CA result in Doppler shifts multiple of $25 \mathrm{~Hz}$, the pre-processing corrects the Doppler. The corrected L1CA Doppler is also shown in Fig. 3. It has significant noise due to integration of I and Q with significant frequency shift in RO receiver. The tracking errors of L2P Doppler, in most cases, cannot be corrected (though, at CDAAC, the correction of the full cycle ambiguities is applied). The large errors of L2P Doppler impede normal processing by making the ionospheric correction impossible, which results in the loss of about $15-20 \%$ of all F3C occultations acquired with L2P.

The un-encrypted L2C signal (sharing frequency with L2P), first broadcast by PRN 17 in 2005, is currently being broadcast by PRNs $1,5,7,12,15,17,25,29$, and 31 . The Jet Propulsion Laboratory (JPL) has modified the F3C receiver firmware to track L2C in both PLL and OL modes. The first test of $\mathrm{L} 2 \mathrm{C}$ tracking on one of the F3C flight modules was performed in 2008 (Meehan et al. 2008). At the beginning of 2012, the updated modified firmware was uploaded to all flight modules. While Meehan et al. (2008) focused mainly on a description of the new L2C signal 
processing in receiver, the current study is focused more on improvements of RO inversions with $\mathrm{L} 2 \mathrm{C}$.

The stability of L2C PLL tracking in the presence of scintillation is better than that of L2P and even better than that of L1CA (see next section). This allows application of the ionospheric correction for almost all L2C occultations thus increasing the number of processed occultations. Furthermore, concurrent wave optics processing of L1CA and L2C signals allows: (1) reduction in the uncertainty of the retrieved bending angles in the lower troposphere; (2) reduction in the small-scale residual errors of the ionospheric correction in the stratosphere, in some occultations. We present initial results obtained with concurrent wave optical processing of L1CA and L2C, while implementation of these approaches into operational processing requires more effort and will be addressed in the future.

\section{L2C tracking}

L2C-transmitted power is lower than L1CA but higher than L2P. Because L2C is an open signal (with disclosed PRN modulation), it allows tracking by full de-spreading of the spectrum without squaring and aiding by L1CA (as opposed to L2P tracking). L1CA aiding allows the L2P PLL to use a narrower loop bandwidth which improves low SNR tracking. But under high ionospheric dynamics, the L2P loop has insufficient bandwidth to maintain phase lock. L2C signals are processed with better dynamic response due to wider tracking loop bandwidth and some features described below. Figure 4 shows distributions of the mean SNRs (averaged between 60 and $80 \mathrm{~km} \mathrm{HSL}$ ) for all F3C flight modules, for all azimuths of GPS with respect to LEO plane, and for L1CA, L2C, and L2P. Currently, in F3C receivers, L2CA is tracked in PLL mode above the transition point (about $-10 \mathrm{~km}$ HSL, corresponding to about $10 \mathrm{~km}$ HTP). Below the transition point, L2CA is tracked in the model-aided OL mode, similar to L1CA.

L2C is composed of L2CL and L2CM signals that share the same carrier frequency $1.2276 \mathrm{GHz}$, but use different modulations. L2CL and L2CM codes have different lengths $(1,500$ and $20 \mathrm{~ms})$ and the same chipping rate $0.5115 \mathrm{MHz}$. The two codes are interleaved into a combined carrier modulation at $1.023 \mathrm{MHz}$. In the OL tracking mode, the smaller chipping rate (i.e., wider autocorrelation function) of L2C compared to L1CA reduces the loss of SNR due to range modeling errors. While L2CL is a pilot signal, L2CM is modulated by navigation data at $50 \mathrm{~Hz}$ rate. In order to maximize the SNR, the L2CL and L2CM signals are processed in two separate correlators which integrate over the $50 \mathrm{~Hz}$ modulation interval. One correlator uses an in-phase model of the CM code and the other a sign-inverted model. To remove the data modulation, the correlator that yields larger SNR is provided on output and the corresponding CM data modulation sign recorded for output. This method allows 4 quadrant PLL processing, providing a $6 \mathrm{~dB}$ improvement to voltage SNR and dynamic response. The effectiveness of this approach is based on approximate equality of L2CL and L2CM SNRs. This approach is different from removal of the data modulation without a pilot signal where the phase is extracted in 2 quadrants. The errors of the data demodulation with the pilot signal depend only on the noise, while the errors of the 2-quadrant phase extraction depend on both the noise and the signal phase fluctuation. This makes PLL tracking of L2C more stable than of L1CA despite lower SNR and despite the fluctuation of the phase caused by diffraction from sharp structures like E layer, which is stronger at lower frequency. Figure 5 shows an example of a F3C occultation where lock on L1CA is lost due to strong ionospheric scintillation at HSL of about $100 \mathrm{~km}$ (the corrected L1CA Doppler is noisy due to low SNR), while lock on L2C is maintained. Statistical analysis of F3C data shows that PLL tracking failures induced by the ionospheric scintillation, like those in Figs. 3 and 5, occur twice more frequently for L1CA than for L2C.

Another way of evaluation of $\mathrm{F} 3 \mathrm{C} \mathrm{L2C} \mathrm{OL}$ data is comparison of L1CA and $\mathrm{L} 2 \mathrm{C}$ signals represented in the form of the sliding spectrograms in the lower troposphere. This representation is commonly used for visualization of the multitone structure of RO signals. Figure 6 shows the sliding spectrograms for the occultation with sharp top of the boundary layer that results in multipath propagation. The complex RO signals were down-converted with the Doppler model based on orbits and bending angle climatology (Sokolovskiy et al. 2009), which shifts the mean frequency close to zero. The gray scale shows the spectral amplitude in the sliding window of $1.28 \mathrm{~s}(6450-\mathrm{Hz}$ samples) as a function of the frequency and the HSL of the center of the window. Panels A and B show the

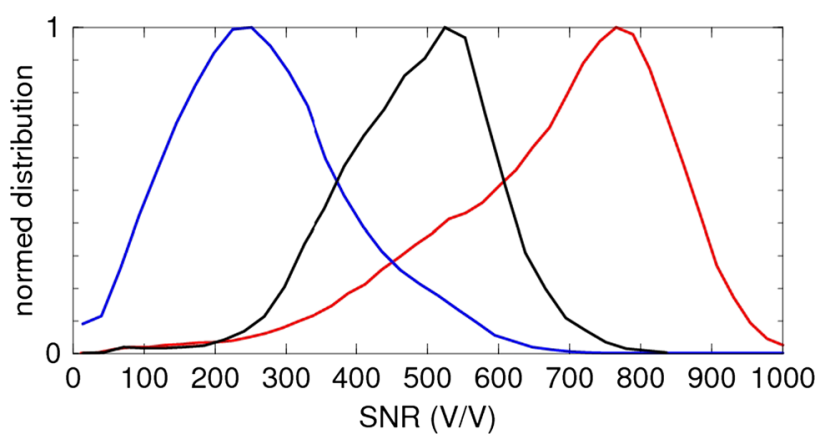

Fig. 4 Distributions of L1CA (red), L2P (blue), and L2C (black) SNRs averaged between HSL 60 and $80 \mathrm{~km}$ 


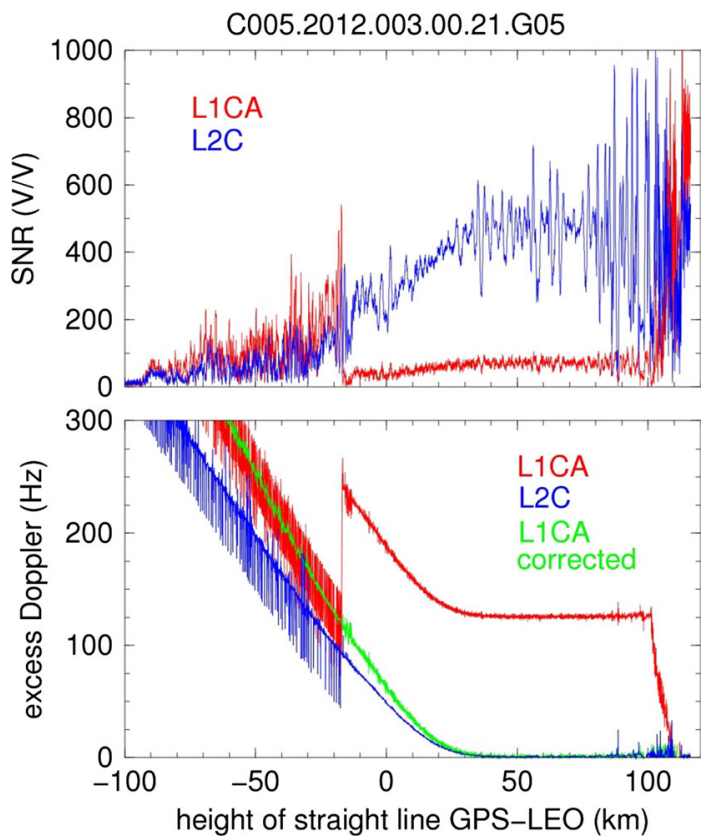

Fig. 5 Example of F3C setting occultation where lock on L1CA is lost due to strong ionospheric scintillation while lock on L2C is maintained. Upper panel L1CA and L2C SNRs; lower panel excess Doppler values (green line shows corrected L1CA Doppler)

spectrograms of the L1CA signal: with external removal of the navigation data modulation by use of replica (A) and with internal removal based on the sign of $\left(I_{i} I_{i+1}+\right.$ $Q_{i} Q_{i+1}$ ) (which is similar to 2-quadrant phase extraction in PLL) (B). Panel C shows the spectrogram of the L2C signal with the removal of data modulation in the receiver aided by pilot signal as discussed above (the frequency separation of multiple sub-signals for L2 is smaller than for L1 due to lower carrier frequency). The multipath propagation in this occultation is well seen in the spectrogram of L1CA with the external removal of data modulation (A) at $-80 \mathrm{~km}<\mathrm{HSL}<-20 \mathrm{~km}$. The internal removal of data modulation (B) smears the multipath structure and artificially de-spreads the spectrum in the noisy part of the signal at HSL $<-130 \mathrm{~km}$ (Sokolovskiy et al. 2009). The removal of data modulation aided by pilot signal (C) allows reproduction of the multipath structure with L2C almost as well as with L1CA with the external removal of data modulation (A). This provides additional proof of the efficiency of removal of data modulation from L2CM aided by L2CL applied in F3C receivers.

A better approach to remove the $\mathrm{L} 2 \mathrm{C}$ data modulation would be to first provide $I$ and $Q$ for $\mathrm{L} 2 \mathrm{CL}$ and $\mathrm{L} 2 \mathrm{CM}$ separately on output from the receiver and post-process them with a replica of the L2CM data modulation. This approach, however, would increase the data volume and

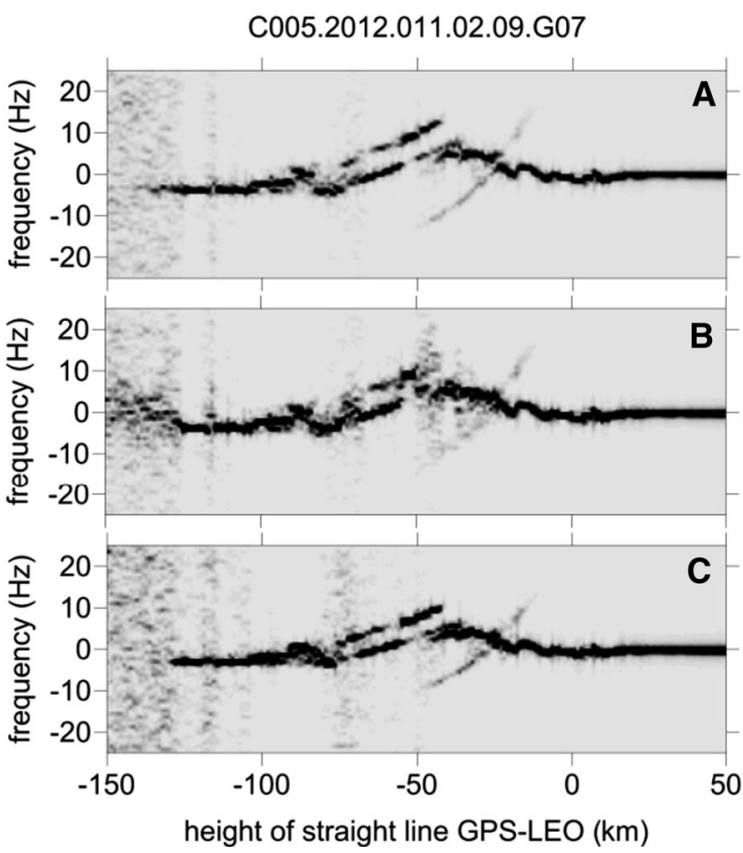

Fig. 6 Sliding spectrograms of the down-converted RO signals for one $\mathrm{F} 3 \mathrm{C}$ tropical occultation. $\mathbf{a}$ and $\mathbf{b}$ L1CA signal with data demodulation by use (a) and without use (b) of the replica. c L2C signal with data demodulation as discussed in Sect. 2

require L2CM data modulation replica, but may be considered for future data processing architecture.

\section{Improvement of current $\mathrm{RO}$ inversions in the neutral atmosphere with L2C}

Improvement of the current CDAAC RO processing in the neutral atmosphere with the use of $\mathrm{L} 2 \mathrm{C}$ comes mainly from the increase in the number of $\mathrm{RO}$ soundings that pass the L2 signal quality control (QC) check. At CDAAC, the following criterion is used to evaluate the quality of L2 signal: the difference between raw and 1-s-smoothed L2 Doppler values exceeds $6 \mathrm{~Hz}$ or the difference between 1-s-smoothed L2 and L1 (scaled by the ratio of L2-L1 frequencies) Doppler values exceeds $1 \mathrm{~Hz}$. This semiempirical criterion was found to satisfactorily detect L2 tracking errors similar to those shown in Fig. 1 and, all the more, in Figs. 2 and 3. The maximal height above the transition point and below $40 \mathrm{~km}$ where this criterion is satisfied is called the L2 drop height. Below this height, the standard ionospheric correction of the bending angles is replaced with a correction of $\mathrm{L} 1$ bending angle by the difference of L1 and L2 bending angles extrapolated from above (Kuo et al. 2004). If the L 2 drop height is found to be larger than $20 \mathrm{~km}$, the occultation is discarded (because of large errors of the extrapolated ionospheric correction). 

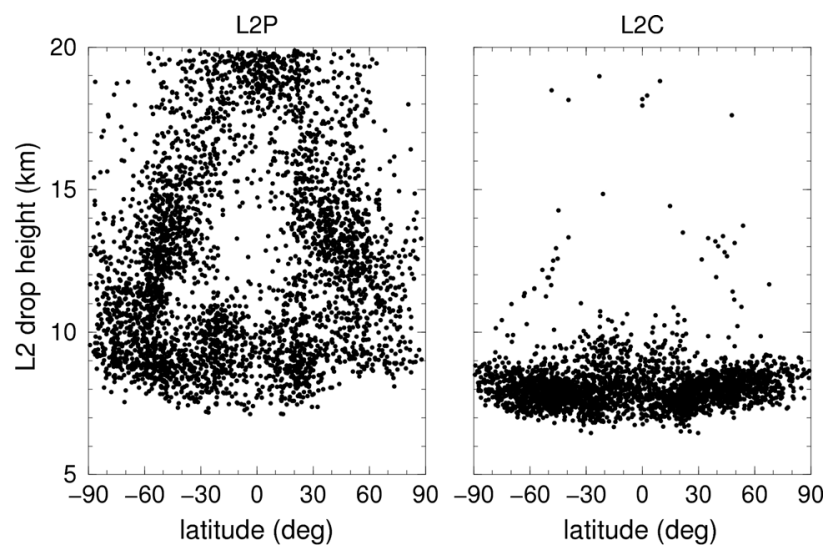

Fig. 7 Scatter plots of L2P (left) and L2C (right) drop heights versus latitudes (for details see text)

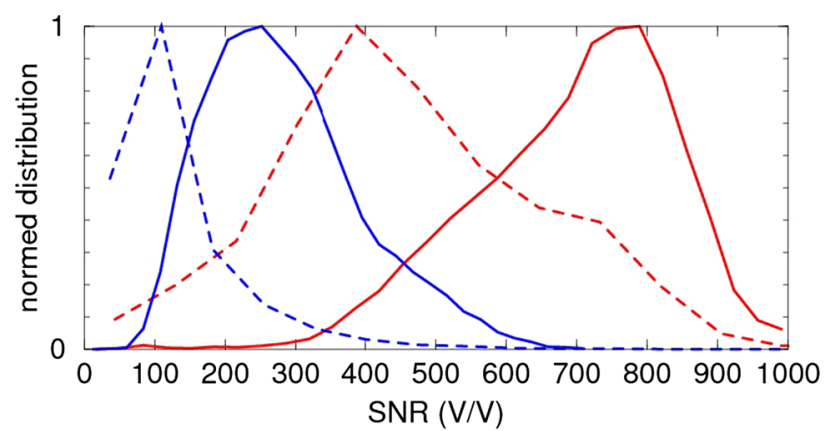

Fig. 8 Distributions of the SNRs (averaged between 60 and $80 \mathrm{~km}$ ) for L1CA (red) and L2P (blue) for the occultations that passed QC (solid lines) and stopped by QC (dashed lines)

Figure 7 shows distributions of F3C L2 drop heights versus latitude for occultations with L2P (left panel) and L2C (right panel) from 2012.001-068. For L2P, the distribution of drop height has two branches. The upper branch follows the latitudinal dependence of the tropopause height (varying from about $20 \mathrm{~km}$ in tropics to about $10 \mathrm{~km}$ in high latitudes), while the lower branch is seen mainly in tropics, at a height of about $10 \mathrm{~km}$. Such distribution of L2P drop heights indicates that sharp structure of the tropopause and the structure of tropical moisture (even as high as at about $10 \mathrm{~km}$ ) result in phase and amplitude fluctuations sufficient to cause tracking instability of L2P. For L2C, the tropopause- and moistureinduced drop heights are rare and the distribution is mainly related to the transition between PLL and OL, which confirms the stability of L2C PLL tracking.

For L2P, $15.7 \%$ of occultations were discarded due to drop heights above $20 \mathrm{~km}$. For L2C, only $1.2 \%$ of occultations were discarded. Figure 8 shows distributions of L1CA and L2P SNRs for the occultations that do pass and do not pass the QC. The occultations with low SNR are more susceptible to the ionospheric scintillation that results in L2P tracking errors. Figure 9 shows standard deviations

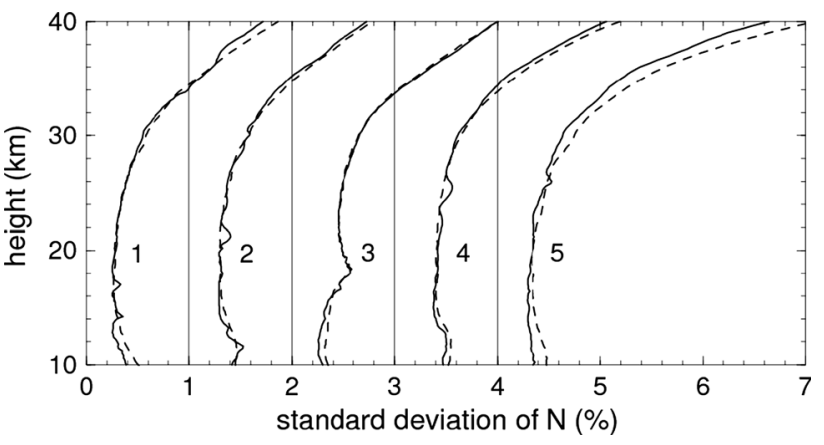

Fig. 9 Vertical profiles of the standard deviations between COSMIC and ECMWF refractivities for L2P (dashed lines) and L2C (solid lines) occultations between Jan 1 and March 31, 2012, for different latitude bands: 60S-90S (1); 30S-60S (2); 30S-30 N (3); $30 \mathrm{~N}-60 \mathrm{~N}$ (4); and $60 \mathrm{~N}-90 \mathrm{~N}$ (5). The profiles are shifted horizontally for better visualization

of RO-retrieved refractivities from those calculated from the ECMWF (European Centre for Medium Range Weather Forecast) model in different latitude bands, for $\mathrm{L} 2 \mathrm{P}$ and $\mathrm{L} 2 \mathrm{C}$ occultations. As seen, the reduction in the standard deviation for L2C compared to L2P is small (mainly seen in the northern high latitudes). This is not surprising, because the occultations that are discarded from the L2P statistic due to tracking errors do contribute to the L2C statistic with their large noise on L1CA. Thus, the main improvement from the use of $\mathrm{L} 2 \mathrm{C}$ in current $\mathrm{RO}$ processing is the increase in the number of occultations that pass QC, while improvement of the quality (reduction in noise) above about $10 \mathrm{~km}$ will be possible in the future, with OL tracking of L1CA and L2C for the whole occultation. The processing method to improve RO inversions below about $10 \mathrm{~km}$ (where L2C currently is tracked in OL mode) is discussed in Sect. 4.

\section{Use of L1CA and L2C for wave optics inversions of bending angles in the lower troposphere}

It is known that the bending angle retrieved from an RO signal by wave optics (WO) methods (which transform RO signal from time/coordinate to impact parameter representation (Gorbunov 2002; Jensen et al. 2003, 2004; Gorbunov and Lauritsen 2004)) has significant uncertainty in the moist troposphere (Sokolovskiy et al. 2010). One of the reasons is the effect of non-spherically symmetric irregularities of refractivity. For a spherically symmetric refractivity, even under severe multipath in the time/coordinate representation, the signal is quasi-monochromatic in the impact parameter representation. In this case, the bending angle calculated by differentiation of the phase has very small uncertainty. In the presence of non-spherically 

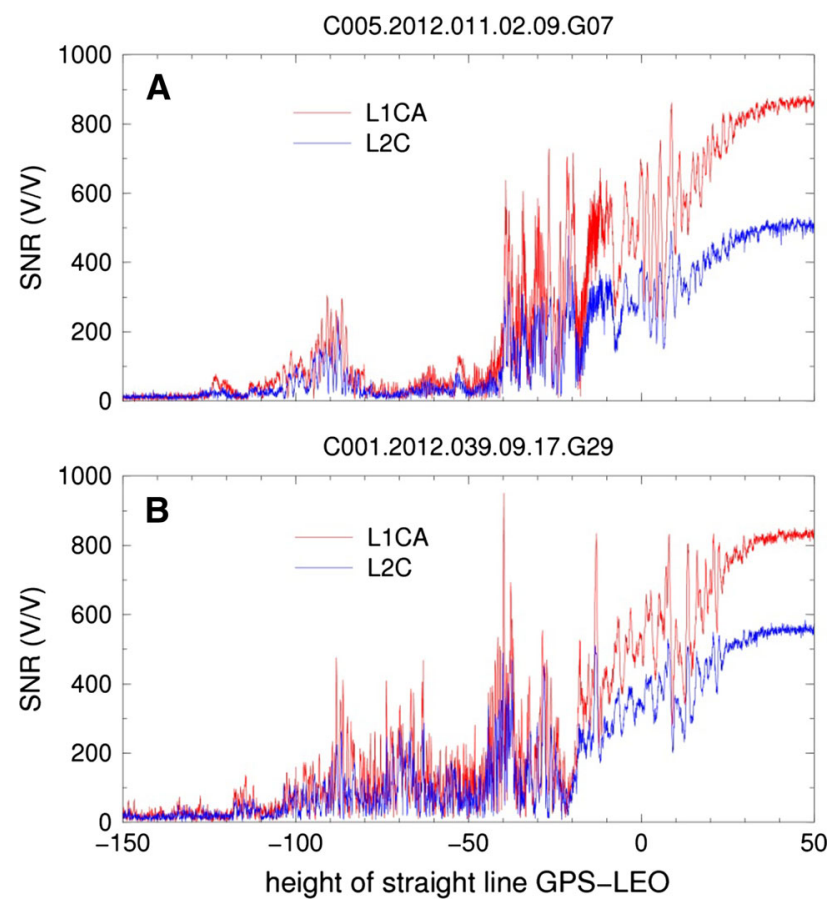

Fig. 10 L1CA and L2C SNRs for two F3C setting tropical occultations

symmetric irregularities, the signal transformed to the impact parameter representation has multitone structure. This results in fluctuations of the amplitude and phase, where the strongest fluctuations of the frequency (contributing to the bending angle uncertainty) correspond to the regions with minimum amplitude.

Figure 10 shows the L1CA and L2C SNRs for two tropical occultations: one with a sharp top of the boundary layer (A) and another one with much deeper layer of strong convection without a sharp top (B). Figure 11 shows the bending angles as functions of the impact height retrieved by Phase Matching (Jensen et al. 2004) separately from L1CA and L2C complex RO signals (the bending angles are smoothed with about $0.1 \mathrm{~km}$ window). The largest random differences between L1CA and L2C bending angles are observed in the regions where local spectra of the WO-transformed RO signals are broad due to the effect of moist convection: below about $3.5 \mathrm{~km}(\mathrm{~A})$, and below $7-8 \mathrm{~km}$ (B). Figure 12 shows the raw bending angles (without smoothing) and the corresponding amplitudes of WO-transformed L1CA and L2C signals for the occultation with deep convection (lower panels are zoomed fragments of the upper panels). It is seen that the largest spikes in the bending angles correspond to the lowest amplitudes. It is also seen that the lowest amplitudes and the corresponding spikes of the bending angles $\alpha$ are observed at different impact heights $h$ at L1 and L2 frequencies. This can be explained by the difference of the diffraction effects at the
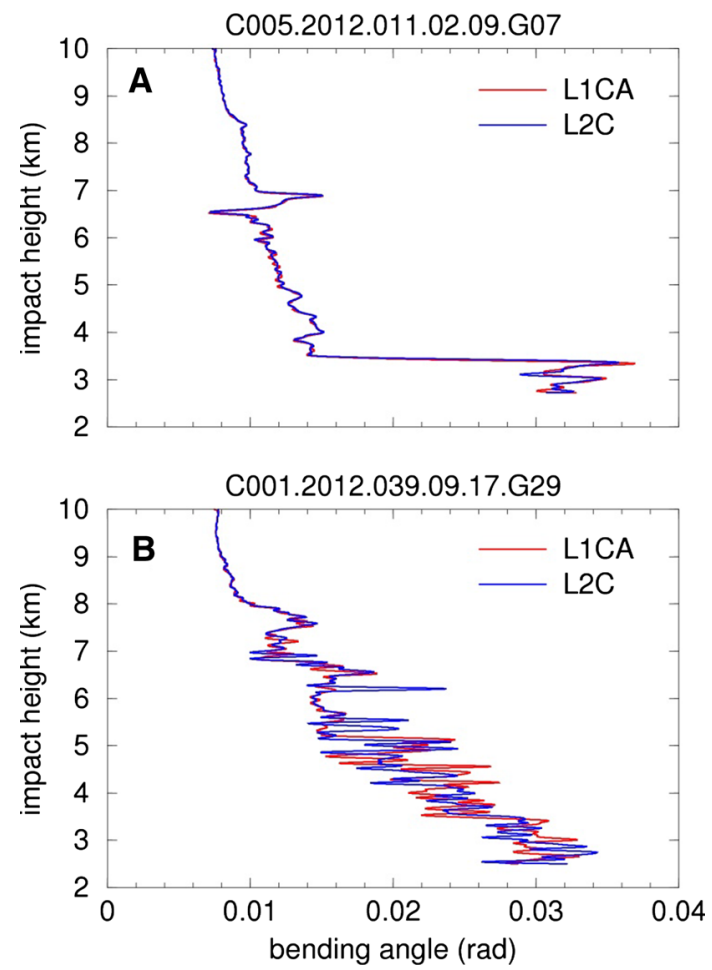

Fig. 11 Bending angles retrieved by the Phase Matching (smoothed with window $0.1 \mathrm{~km}$ ) from L1CA and L2C signals independently, for the two tropical occultations (SNRs shown in Fig. 10)

L1 and L2 frequencies, which manifests itself mainly in the small-scale variations (larger-scale variations are more correlated). This permits the following approach for the calculation of neutral atmospheric bending angle from L1 and L2 bending angles $\alpha_{1}(h)$ and $\alpha_{2}(h)$ by utilizing the WO-transformed amplitudes $B_{1}(h)$ and $B_{2}(h)$ :

$\alpha(h)=\left\{\begin{array}{lll}\alpha_{1}(h)+\alpha_{\text {ion }} f_{2}^{2} /\left(f_{1}^{2}-f_{2}^{2}\right) & \text { when } & B_{1}(h)>B_{2}(h) \\ \alpha_{2}(h)+\alpha_{\text {ion }} f_{1}^{2} /\left(f_{1}^{2}-f_{2}^{2}\right) & \text { when } & B_{2}(h)>B_{1}(h)\end{array}\right.$

where the ionosphere correction term $\alpha_{\text {ion }}=<\alpha_{1}(h)-$ $\alpha_{2}(h)>$ is averaged in a certain height range above the troposphere (this is a commonly applied "extrapolation" of the ionospheric correction in the troposphere mentioned in Sect. 3). This approach can be called comparative discrimination, similarly to discrimination by thresholding applied in digital signal processing (Sirmans and Bumgarner 1975). Besides the L1 and L2 bending angles, Fig. 12 also shows the combined bending angle $\alpha(h)$ where the spikes are eliminated or substantially reduced. Figure 13 shows the bending angles $\alpha(h)$ smoothed with $0.1 \mathrm{~km}$ window and the responses of the smoothed $\alpha_{1}(h)$, $\alpha_{2}(h)$, and $\alpha(h)$ to additive noise imposed on the observed complex RO signals [this test of the response to additive noise is the same as applied in Sokolovskiy et al. (2010)]. It is seen that combining the L1 and L2 bending angles with 

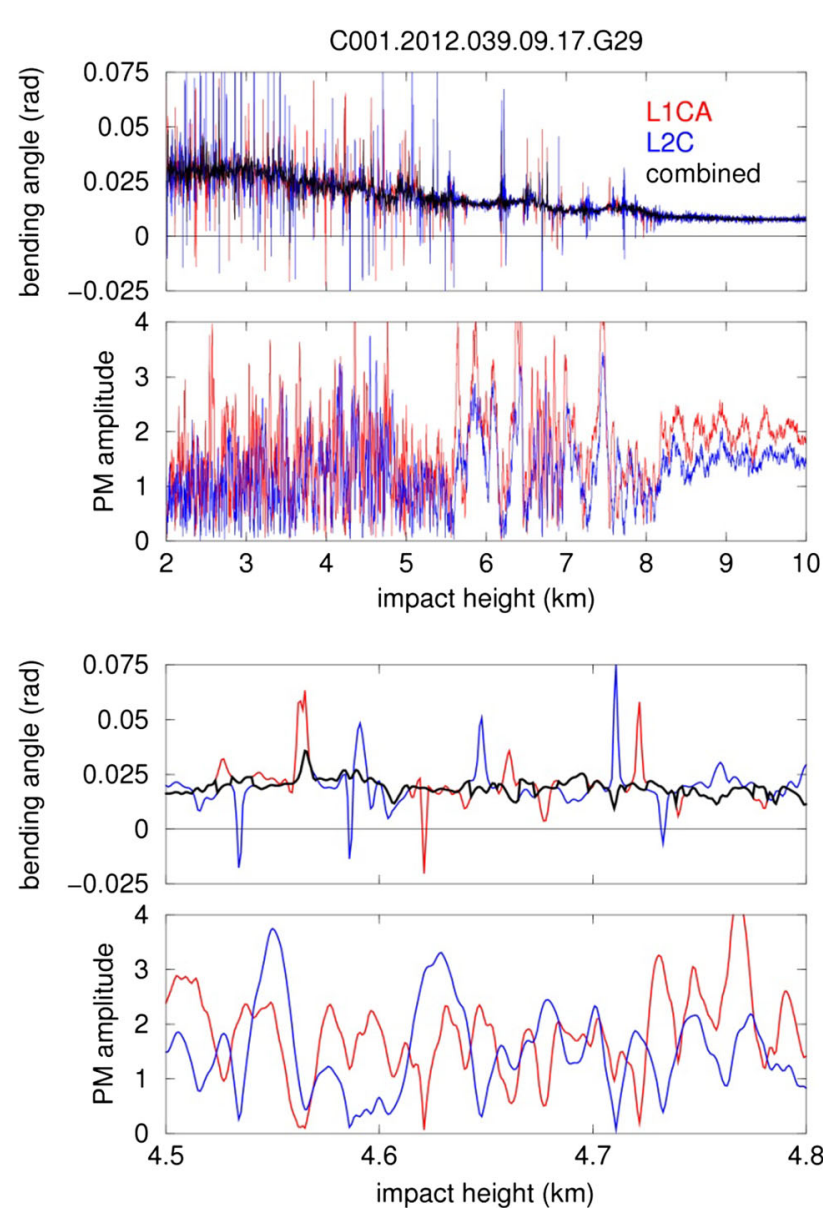

Fig. 12 Bending angles and amplitudes calculated from complex RO signal transformed to impact height representation by the Phase Matching for one F3C tropical occultation. Red and blue lines show bending angles calculated separately from L1CA and L2C signals (no smoothing); black line shows combined bending angle (for details see text)

use of the WO-transformed amplitudes substantially reduces the sensitivity of the resulting bending angle to noise. We note that simple summation of L1 and L2 bending angles does not reduce the magnitude of the spikes and the sensitivity to noise. Thus the approach introduced above reduces the uncertainty of $\mathrm{RO}$ in the moist lower troposphere. Validation of this approach by routine processing of large amounts of data will be addressed in the future.

\section{Reduction in ionospheric correction errors by back propagation of L1CA and L2C signals}

Dual frequency model-independent ionospheric correction of bending angles applied in GPS RO has both large- and small-scale residual errors that represent a dominant error source of RO in the middle and upper stratosphere (above about $30 \mathrm{~km}$ ). The large-scale errors mainly arise from the separation of rays at $\mathrm{L} 1$ and $\mathrm{L} 2$ frequencies and from the higher order terms of the dependence of refractivity on frequency (Hardy et al. 1993; Syndergaard 2000). Mitigation of the large-scale errors is outside the scope of this study. The small-scale errors (with scales of order of $1 \mathrm{~km}$ or less) have much larger magnitude and mainly arise from diffraction effects that are different at L1 and L2 frequencies, and from the ray separation as well. Below, we consider mitigation of the diffraction effects.

Since the diffraction effects increase with propagation distance, they may be reduced by propagation of the complex electromagnetic field from the observational trajectory back to the location of the ionospheric irregularities. Such back propagation (BP) of the $50-\mathrm{Hz}$ sampled L1CA signal has been applied previously for localization of the ionospheric irregularities (Gorbunov et al. 2002; Sokolovskiy et al. 2002).

To make use of BP, the irregularities must satisfy the following conditions: they must occupy a volume much smaller than the propagation distance and they must be anisotropic (elongated) with known orientation. The latter condition is necessary because RO signal is measured on a $1 \mathrm{D}$ trajectory and thus only $2 \mathrm{D}$ (not $3 \mathrm{D}$ ) propagation can be applied. The BP plane must be normal to projections of the irregularities on the plane normal to propagation direction. The receiver trajectory is projected on the BP plane by assuming stationary transmitter. Then, the location of the minimum of the amplitude fluctuation of the BP field corresponds to the location of the irregularities. Ionospheric correction of the bending angles calculated from BP field at the location of irregularities must have minimal residual errors related to diffraction effects on L1 and L2 frequencies. When the orientation of the irregularities is not known, then the location of the minimum of the amplitude fluctuation is different from the true location of the irregularities, but the ionospheric correction at the location of the minimum of amplitude fluctuation still results in the minimal errors related to diffraction effects. This was confirmed by modeling the $2 \mathrm{D}$ wave propagation at two frequencies (not shown).

While BP of L1CA is feasible, BP of L2P, encrypted by $\mathrm{Y}$-code and tracked in semi-codeless mode, is not feasible in most cases affected by scintillation due to large tracking errors. In Gorbunov et al. 2002, the BP of L2P was applied for the data obtained in GPS/MET mission during limited periods (prime times) when L2P encryption was temporary turned off. Transmission of L2C allows application of BP for both $\mathrm{L} 1$ and $\mathrm{L} 2$ for the purpose of reduction in the diffraction-induced errors of the ionospheric correction.

Technical details of BP, including calculation of the virtual positions of GPS and LEO (to make transmitter stationary) and projection of the virtual LEO trajectory on the BP plane with arbitrary orientation, were discussed in 

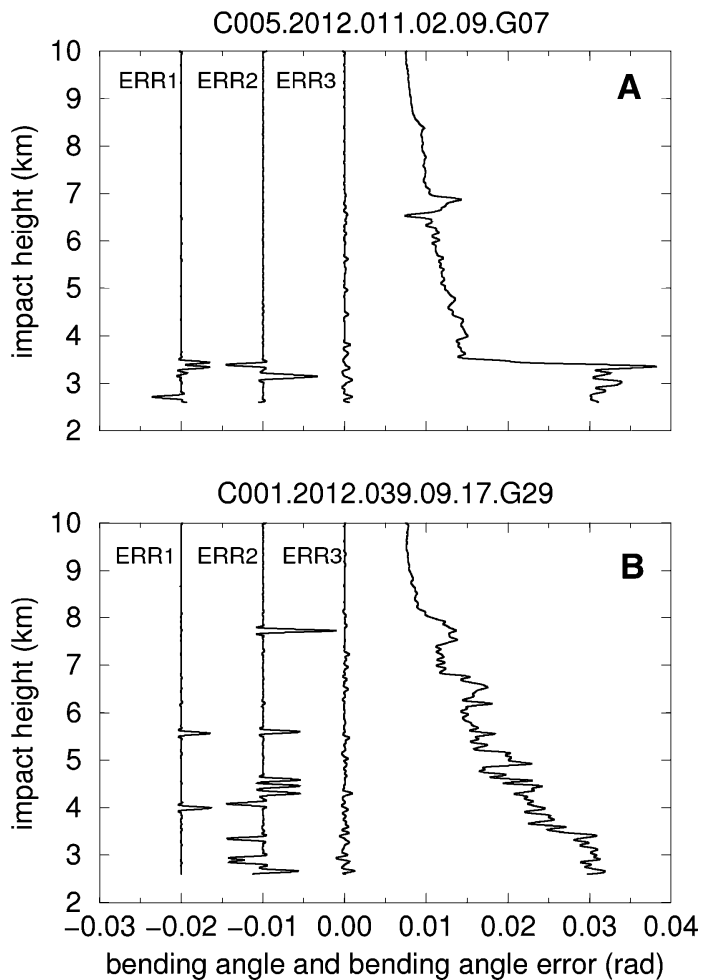

Fig. 13 Bending angles and their response to noise for two F3C tropical occultations (a, b). The bending angles (right curves) were retrieved by combining WO-transformed L1CA and L2C signals. Other curves show response of the bending angles to additive noise on complex RO signals: of the L1CA bending angle (ERR1, shifted by $0.02 \mathrm{rad}$ ); of the L2C bending angle (ERR2, shifted by $-0.01 \mathrm{rad})$; of the combined bending angle (ERR3)

Sokolovskiy et al. (2002). As mentioned above, for the purpose of the ionospheric correction, there is no need to account for the orientation of irregularities, and in this study, we project the virtual LEO trajectory on a vertical BP plane (thus assuming that the irregularities are aligned horizontally).

Figure 14 shows L1CA and L2C SNRs for four F3C occultations affected by ionospheric scintillation. Classification of the ionospheric effects on the amplitude and phase of RO signals can be found in Pavelyev et al. (2005). While extended scintillation with uniform structure (B) is more common for the $\mathrm{F}$ layer, scintillation producing isolated U-shaped structures in amplitude $(\mathrm{C}, \mathrm{D})$ is common for the sporadic E layer (or E-clouds) (Zeng and Sokolovskiy 2010). The origin of the ionospheric scintillation in case (A) is less obvious. Figure 15 shows the results of BP of L1CA and L2C signals for the four selected occultations. The horizontal axis is the distance along the line connecting GPS (on the left) and LEO (on the right) at a certain HSL (about $30 \mathrm{~km}$ ), counted from the tangent point. The vertical axis is normal to the horizontal, counted from the line touching the earth's surface. The fluctuating
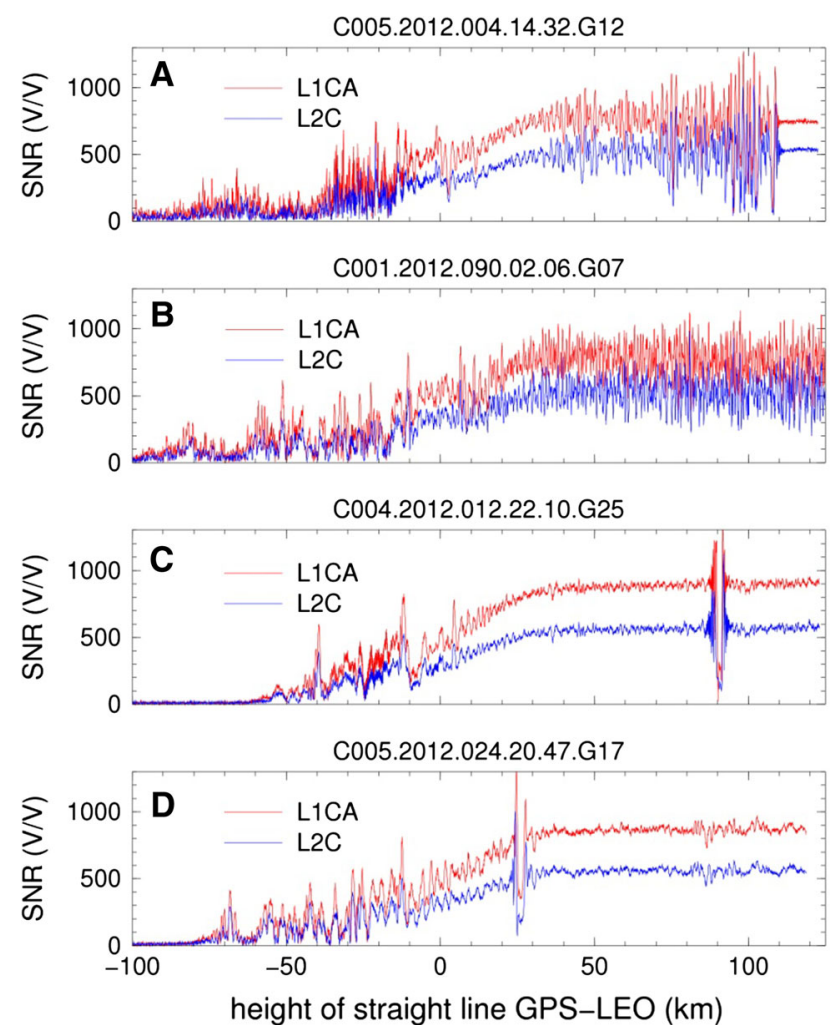

Fig. 14 L1CA and L2C SNRs for four F3C occultations affected by the ionospheric scintillation. The source of the scintillation a is not obvious. The scintillation $\mathbf{b}$ comes from $\mathrm{F}$ layer, and the scintillation in the form of U-shaped structures (c, d) from E layer

vertical lines denote, in a certain scale, amplitude fluctuation of the signal, while their mean horizontal positions correspond to those locations where the signals were calculated by BP. It is seen that there are regions where amplitude fluctuations of the BP signals are minimal. These correspond to horizontal distances: about $-1,000 \mathrm{~km} \mathrm{(A),}$ $+1,000 \mathrm{~km}(\mathrm{~B}), 0 \mathrm{~km}(\mathrm{C})$, and $-800 \mathrm{~km}(\mathrm{D})$. We note that in case (A), the distance to the region of minimal amplitude fluctuation remains about $-1,000 \mathrm{~km}$ up to a height of about $80 \mathrm{~km}$, then changes to about $-200 \mathrm{~km}$ at a height of about $100 \mathrm{~km}$. For the neutral atmospheric inversions, the bending angles above $80 \mathrm{~km}$ are not used; thus, we use the distance $-1,000 \mathrm{~km}$. If the ionospheric irregularities are aligned horizontally, the regions of the minimal amplitude fluctuations approximately represent the true locations of irregularities. As discussed above, to calculate the L1 and L2 bending angles for the purpose of the ionospheric correction, we use the $\mathrm{BP}$ signals from the regions of the minimal amplitude fluctuations regardless of whether they represent the true locations of irregularities or not.

Figure 16 shows the bending angles as functions of impact heights calculated by geometric optics with smoothing. Red and blue lines correspond to L1CA and L2C bending angles calculated without $\mathrm{BP}$, and black 
Fig. 15 Amplitudes of backpropagated complex RO signals for the four F3C occultations (SNRs shown in Fig. 14) affected by the ionospheric scintillation (for details see text)
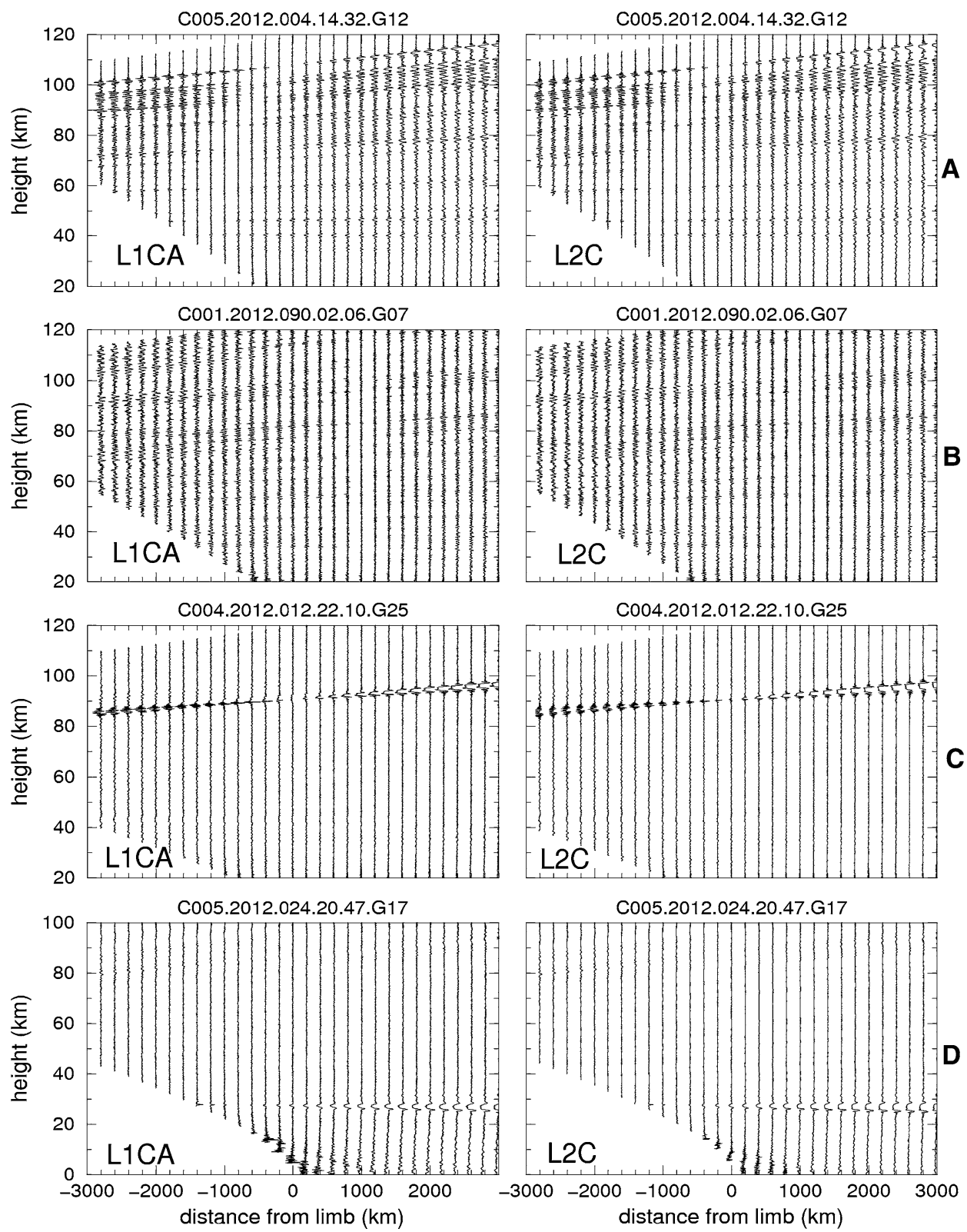

dashed lines are their "ionosphere-free" linear combinations. The black solid lines show the "ionosphere-free" linear combinations of the bending angles calculated from RO signals after BP to the locations of minimum amplitude fluctuations (the distances were indicated above). It is seen that for all selected occultations affected by the ionospheric scintillations, the BP approach reduces the diffractioninduced errors of the ionospheric correction. The most significant error reduction is found in cases of the scintillation induced by sporadic E layer (C,D). When the effect of $\mathrm{E}$ layer is observed at the tangent heights where bending angle is not used for the neutral atmospheric inversions (C), the error reduction is not so important. However, it is important at lower heights, where the effect of tilted E layer results in significant inversion errors (D) that can be reduced by applying BP. Analysis of individual occultations affected by ionospheric scintillation shows that the fluctuation of BP amplitude does not always have a clear minimum. In some cases, the minimum does exist, but its horizontal location depends on height (as in case (A) in Fig. 15). Thus, reduction in the errors of ionospheric correction may not be possible for all occultations. To evaluate error reduction in a statistical sense, processing of a large amount of data with an automated search for the minima of the fluctuation of BP amplitude is needed; this will be addressed in the future.

Our testing shows that application of WO methods (Gorbunov 2002; Jensen et al. 2003, 2004; Gorbunov and Lauritsen 2004) for calculation of bending angles yields similar reduction in the ionospheric correction errors as 
Fig. 16 Bending angles for the four $\mathrm{F} 3 \mathrm{C}$ occultations affected by the ionospheric scintillation (SNRs shown in Fig. 14, BP amplitudes in Fig. 15).

Calculated by GO: L1CA (red); L2C (blue); ionosphere free (black dashed). Calculated by GO after BP: ionosphere free (black solid)
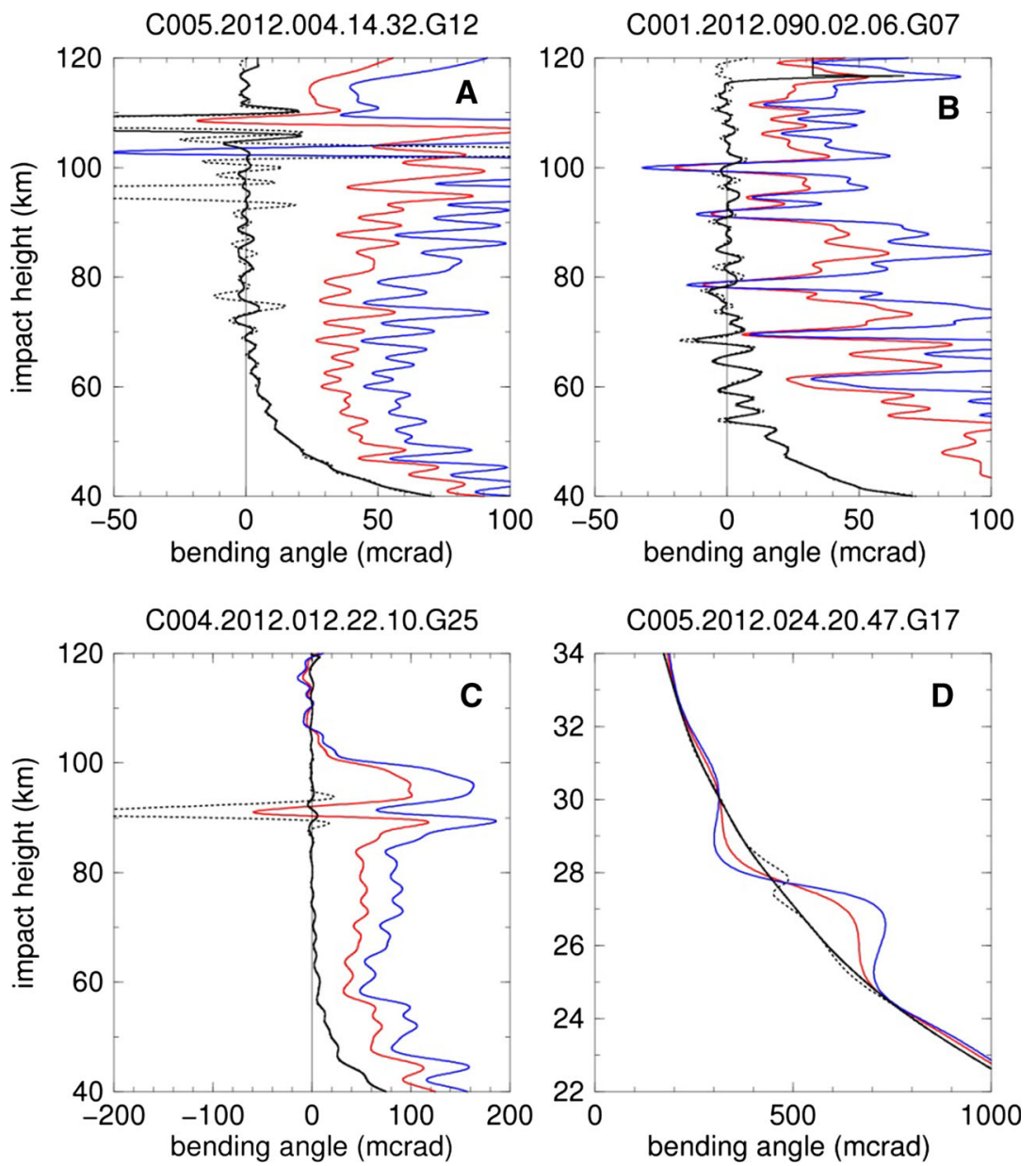

$\mathrm{BP}+\mathrm{GO}$ when fluctuation of $\mathrm{BP}$ amplitude has minimum at a distance close to zero (i.e., at the limb). If the minimum is far away from the limb, the error of the ionospheric correction of WO-calculated bending angles is larger than of those calculated by $\mathrm{BP}+\mathrm{GO}$. This is related to uncertainty of the bending angles calculated by WO methods for non-spherically symmetric refractivity. For large-scale irregularities, this uncertainty has been demonstrated in Gorbunov et al. (2010).

\section{Conclusions}

Current RO processing is substantially affected by PLL tracking errors on L2P (to a lesser extent on L1CA) signals caused by ionospheric scintillation and low SNR. At CDAAC, due to the inability to apply an ionospheric correction because of corrupted L2P signal, about 15-20\% of the occultations with L2P are discarded.

The new L2C signal has PLL tracking stability in the presence of scintillation that is better than L2P and even better than L1CA. Also, L2C allows OL tracking for the whole occultation. $\mathrm{F} 3 \mathrm{C}$ receivers were modified to track
L2C signals from beginning January 1, 2012. Currently, both L1CA and L2C are tracked in OL mode in the troposphere (below about $10 \mathrm{~km}$ ) and in PLL mode above. This results in processing of about $100 \%$ of occultations from the PRNs with available L2C (compared to about 80-85\% with L2P), thus increasing the total number of processed occultations. The quality of the refractivity profiles retrieved with $\mathrm{L} 2 \mathrm{C}$ is not substantially improved in statistical sense. This is explained by extra noise on L1CA Doppler due to ionospheric scintillation and low SNR for those occultations that would not pass QC due to tracking errors if processed with L2P.

In the future, improvement of the quality of refractivity profiles retrieved with the use of $\mathrm{L} 2 \mathrm{C}$ is possible in two ways: in the receiver and in the inversions. In the receiver, increasing the antenna gain and/or applying OL tracking for L1CA and L2C for the whole occultation will eliminate tracking errors. In the inversion process: (1) combining L1CA and L2C in wave optics inversions in the troposphere reduces the uncertainty of the retrieved bending angle; and (2) reduction in the diffractional effects on L1CA and L2C signals by BP reduces the small-scale residual errors of the ionospheric correction for the 
occultations affected by ionospheric scintillation. Both approaches, which are introduced and tested by individual occultations in this study, will be applied and tested in routine $\mathrm{RO}$ processing in the future.

Acknowledgments Research performed at the University Corporation for Atmospheric Research was supported by the National Science Foundation under the Cooperative Agreement AGS-1033112. Portions of this research were carried out at the Jet Propulsion Laboratory of the California Institute of technology, under a contract with the National Aeronautics and Space Administration.

Open Access This article is distributed under the terms of the Creative Commons Attribution License which permits any use, distribution, and reproduction in any medium, provided the original author(s) and the source are credited.

\section{References}

Ao CO, Hajj GA, Meehan TK, Dong D, Ijima BA, Mannucci AJ, Kursinski ER (2009) Rising and setting GPS occultations by use of open-loop tracking. J Geophys Res 114:D04101. doi:10.1029/ 2008JD010483

Gorbunov ME (2002) Canonical transform method for processing radio occultation data in the lower troposphere. Radio Sci 37(5):1076. doi:10.1029/2000RS002592

Gorbunov ME, Lauritsen KB (2004) Analysis of wave fields by Fourier integral operators and their application for radio occultations. Radio Sci 39:RS4010. doi:10.1029/2003RS002971

Gorbunov ME, Gurvich AS, Shmakov AV (2002) Back-propagation and radio-holographic methods for investigation of sporadic ionospheric E-layers from Microlab-1 data. Int J Remote Sensing 23(4):675-685

Gorbunov ME, Lauritsen KB, Leroy SS (2010) Application of Wigner distribution function for analysis of radio occultations. Radio Sci 45:RS6011. doi:10.1029/2010RS004388

Hardy KR, Hajj GA, Kursinski ER (1993) Accuracies of atmospheric profiles obtained by GPS radio occultations. Proceedings on ION-GPS-1993, Institute of Navigation, September 22-24, Salt Lake City, UT, pp 1545-1556

Jensen AS, Lohmann MS, Benzon H, Nielsen AS (2003) Full spectrum inversion of radio occultation signals. Radio Sci 38(3):1040. doi:10.1029/2002RS002763

Jensen AS, Lohmann MS, Nielsen AS, Benzon H (2004) Geometric optics phase matching of radio occultation signals. Radio Sci 39:RS3009. doi:10.1029/2003RS002899

Kuo Y-H, Wee T-K, Sokolovskiy S, Rocken C, Schreiner W, Hunt D, Anthes RA (2004) Inversion and error estimation of GPS radio occultation data. J Meteor Soc Jpn 82(1B):507-531

Kursinski ER, Hajj GA, Schofield JT, Linfield RP, Hardy KR (1997) Observing Earth's atmosphere with radio occultation measurements using the global positioning system. J Geophys Res 102(D19):23429-23465

Meehan T, Brooks T, Young L (2000) P-Code enhanced method for processing encrypted GPS signals without knowledge of the encryption code. U.S. Patent No. 6,061,390

Meehan TK, Ao CO, Ijima B, Robinson D, Hunt D, Rocken C, Schreiner W, Sokolovskiy S (2008) A demonstration of L2C tracking from space for atmospheric occultation. In: Proceedings on ION GNSS 2008, Institute of Navigation, September 16-19, 2008, Savannah, GA, pp 698-701
Pavelyev AG, Wickert J, Liou YA, Reigber Ch, Schmidt T, Igarashi K, Pavelyev AA, Matyugov SS (2005) Different mechanisms of the ionospheric influence on GPS occultation signals. GPS Solutions 9(2):96-104

Sirmans D, Bumgarner B (1975) Numerical comparison of five mean frequency estimators. J Appl Meteor 14:991-1003

Sokolovskiy S (2001) Tracking tropospheric radio occultation signals from low earth orbit. Radio Sci 36(3):483-498

Sokolovskiy S, Rocken C (2004) Method and system for determining the phase and amplitude of radio occultation signal. U.S. Patent No. 6,731,906

Sokolovskiy S, Schreiner W, Rocken C, Hunt D (2002) Detection of high-altitude ionospheric irregularities with GPS/MET. Geophys Res Lett 29:3. doi:10.1029/2001GL013398

Sokolovskiy S, Rocken C, Schreiner W, Hunt D, Johnson J (2009) Postprocessing of L1 GPS radio occultation signals recorded in open-loop mode. Radio Sci 44:RS2002. doi:10.1029/2008R S003907

Sokolovskiy S, Rocken C, Schreiner W, Hunt D (2010) On the uncertainty of radio occultation inversions in the lower troposphere. J Geophys Res 115:D22111. doi:10.1029/2010JD014058

Syndergaard S (2000) On the ionosphere calibration in GPS radio occultation measurements. Radio Sci 35(3):865-883

Woo KT (2000) Optimum semi-codeless carrier phase tracking of L2 Navigation. J Inst Navig 47(2):82-99

Zeng Z, Sokolovskiy S (2010) Effect of sporadic E clouds on GPS radio occultation signals. Geophys Res Lett 37:L18817. doi:10. 1029/2010GL044561

\section{Author Biographies}

Dr. Sergey V. Sokolovskiy is a project scientist in COSMIC Program at the University Corporation for Atmospheric Research. Currently, his research is focused on different aspects of the GPS radio occultation remote sensing of the neutral atmosphere.

Dr. William S. Schreiner is the manager of the COSMIC (Constellation Observing Systems for Meteorology, Ionosphere, and Climate) Data Analysis and Archive Center (CDAAC) at UCAR. He possesses experience and knowledge of the algorithms and geodetic modeling used for precise orbit and clock determination of low earth orbiting (LEO) receivers, ground-based position and zenith tropospheric delay estimation, GPS clock offset estimation, derivation of atmospheric excess phase (the fundamental observable of radio occultation), and computation of absolute total electron content (TEC). Also, he has detailed understanding of algorithms used for both neutral atmospheric and ionospheric inversions, and experience in RO payload and mission design for the GPS/MET, COSMIC, and COSMIC-2 missions.

Dr. Zhen Zeng is a project scientist in COSMIC Program at the University Corporation for Atmospheric Research. Currently, her research interests are focused on the evaluation, optimization, and development of inversion algorithms for GPS radio occultation (RO) measurements, and the application of RO data to the atmospheric science.

Douglas C. Hunt has been a software engineer for 25 years, working first at IBM on satellite data processing systems for the US Air Force and later at UCAR on radio occultation data processing. He is currently a senior software engineer at UCAR and the software architect of the CDAAC radio occultation processing system. His interests include satellite data formats, signal processing, web 
programming, database systems, and perl programming. He has coauthored over 20 papers on radio occultation data processing.

Dr. Ying-Hwa Kuo Director of the COSMIC (Constellation Observing Systems for Meteorology, Ionosphere, and Climate) Program at UCAR, is a Senior Scientist of NCAR. He received his $\mathrm{Ph} . \mathrm{D}$. from the Penn State University in 1983. Dr. Kuo has published more than 160 journal papers. His scientific interest includes mesoscale modeling, hurricanes, mesoscale convective systems, GPS meteorological applications, and data assimilation.

Thomas K. Meehan is a Principal Member of the Technical Staff at JPL. Mr. Meehan has worked on high-precision GPS design and development JPL since 1986. He has led three major GPS instrument developments at JPL. He is currently working with scientists for the GRACE and COSMIC spacecraft to devise improved algorithms for GPS-based occultations and reflections and works as one of the principal designers of the TriG, GNSS space receiver.
Theodore W. Stecheson has worked as a software engineer at Jet Propulsion Laboratory in Pasadena since 1999.

Dr. Anthony J. Mannucci received a Ph.D. in physics from UC Berkeley in 1989. He currently supervises the Ionospheric and Atmospheric Remote Sensing Group at NASA's Jet Propulsion Laboratory in Pasadena, California.

Dr. Chi O. Ao received his A.B. degree in physics from the University of California at Berkeley in 1993 and his Ph.D. in physics from the Massachusetts Institute of Technology in 2001. He has been working in the Tracking Systems and Applications Section at the Jet Propulsion Laboratory in Pasadena, California, since 2001. His research interests include the techniques and climate applications of GPS radio occultations. 